\title{
Influência dos Contextos Escolar e Familiar nos Projetos de Vida de Adolescentes
}

\author{
Bruna Caroline Pereira ${ }^{1}$ \\ Cristian Zanon ${ }^{2}$ \\ ${ }^{1}$ Pontifícia Universidade Católica de Campinas, SP, Brasil. $\quad$ 2Universidade Federal do Rio Grande do Sul, RS, Brasil. \\ Letícia Lovato Dellazzana-Zanon ${ }^{1}$ \\ ${ }^{1}$ Pontifícia Universidade Católica de Campinas, SP, Brasil.
}

\begin{abstract}
Resumo: Considerando que família e escola são os contextos em que o adolescente passa mais tempo, este estudo teve como objetivo compreender as possíveis relações entre os projetos de vida de adolescentes e as variáveis dos contextos escolar e familiar. Participaram 320 adolescentes, de ambos os sexos, de 14 a 17 anos, do 9o ano de Ensino Fundamental II e do Ensino Médio de diferentes tipos de escolas de uma cidade do interior de São Paulo. Utilizou-se os instrumentos Ficha de Dados Sociodemográficos e Escala de Projeto de Vida para Adolescentes. Os dados foram analisados por meio de testes $t$ de student, ANOVA oneway e correlação de Pearson. Os resultados indicaram que adolescentes mais novos têm mais projetos de vida em relação a Relacionamentos Afetivos, Religião/Espiritualidade e Bens Materiais do que os mais velhos. Observou-se uma tendência dos projetos da dimensão Religião/Espiritualidade diminuírem conforme os adolescentes crescem e conforme aumentam a Escolaridade da Mãe e a Escolaridade do Pai. Em relação ao tipo de escola, os adolescentes de escolas públicas têm mais projetos de vida da dimensão Relacionamentos Afetivos do que os adolescentes dos demais tipos. Os projetos da dimensão Religião/ Espiritualidade são maiores nos adolescentes de escolas públicas.
\end{abstract}

Palavras-chave: Projetos de Vida, Adolescência, Escola, Família.

\section{Influence of School and Family Contexts in Adolescents' Life Purposes}

\begin{abstract}
Considering that adolescents spend most of their time with their families and within the school environment, this study aimed to understand possible associations and influences of these contexts in adolescents' life purpose. The sample consisted of 320 adolescents aged between 14 and 17 years old, of both genders, enrolled in the 9th grade of Elementary School II and High School of public and private schools and full-time public schools of a city of the countryside of São Paulo. Data were collected using the Sociodemographic Data Sheet and the Purpose in Life Questionnaire for Adolescents and analyzed using Student's t-tests, one-way ANOVA, and Pearson's correlation coefficient. The results show that the life purpose of younger teens are more oriented towards Affective Relationships, Religion/Spirituality, and Material Goods than that of older ones. Projects related to Religion/Spirituality tend to decrease with increasing age and parental education level. Regarding the type of school, the life purpose of adolescents from public schools is more associated with Affective Relations and Religion/ Spirituality than that of adolescents from private schools.
\end{abstract}

Keywords: Purpose in Life, Adolescence, School, Family. 


\title{
Influencia de los Contextos Escolar y Familiar en los Proyectos de Vida de Adolescentes
}

\begin{abstract}
Resumen: Teniendo en cuenta que la familia yla escuela son los contextos en que los adolescentes pasan la mayor parte del tiempo, este estudio tuvo como objetivo comprender las posibles relaciones entre las variables de los contextos escolares y familiares y los proyectos de vida de los adolescentes. Participaron 320 adolescentes, de ambos sexos, de los 14 a los 17 años de edad, del noveno grado de la escuela primaria II y secundaria de diferentes tipos de escuelas en una ciudad del interior de São Paulo. Los instrumentos fueron Hoja de datos sociodemográficos y Escala de proyectos de vida para adolescentes. Los datos se analizaron mediante pruebas t de Student, ANOVA one-way y correlación de Pearson. Los resultados indicaron que los adolescentes más jóvenes tienen más proyectos de vida con respecto a las relaciones afectivas, la religión/ espiritualidad y los bienes materiales que los mayores. Hubo una tendencia a que los proyectos de la dimensión Religión/Espiritualidad disminuyan a medida que crecen los adolescentes y aumentan la Educación de la madre y la Educación del padre. Con respecto al tipo de escuela, los que estudian en escuelas públicas tienen más proyectos de vida en la dimensión Relaciones afectivas que los adolescentes de otros tipos. Los adolescentes de escuelas públicas son los que tienen más proyectos en la dimensión Religión/Espiritualidad.
\end{abstract}

Palabras clave: Proyectos de Vida, Adolescencia, Escuela, Familia.

\section{Introdução}

A construção do projeto de vida na adolescência é uma das tarefas mais importantes dessa fase do desenvolvimento, pois tem um papel central na orientação dos objetivos fundamentais de um indivíduo (Erikson, 1968/1976). Investigar esse tema é importante, pois a presença de projetos de vida na adolescência está associada a uma série de aspectos positivos para o desenvolvimento (Damon, Menon, \& Bronk, 2003), como envolvimento e contribuição para a comunidade e a sociedade (Johnson, Tirrell, Callina, \&Weiner, 2018) e desenvolvimento de uma vida mais saudável e feliz (McKnight \& Kashdan, 2009). Por outro lado, não ter um projeto de vida pode causar uma série de problemas, como incapacidade de manter uma relação interpessoal estável, sensação de estar à deriva e falta de produtividade (Damon, 1995; Damon et al., 2003).

Sabe-se que o nível socioeconômico tem muita influência na construção do projeto de vida dos adolescentes, visto que as famílias que têm limitações financeiras expressivas geralmente estão inseridas em um contexto desprivilegiado e, por isso, podem vivenciar situações de vulnerabilidade e baixa escolaridade (Dellazzana-Zanon, Riter, \& Freitas, 2015). Em estudo realizado por Cardoso e Cocco (2003) com adolescentes de uma Unidade Básica de Saúde no estado de São Paulo, foi verificado que apesar das dificuldades enfrentadas, os adolescentes de nível socioeconômico baixo tinham um projeto de vida. Observou-se nesse estudo que os projetos de vida dos adolescentes de escolas públicas delineavam "conquistas mais humildes diante das possibilidades oferecidas no seu dia-a-dia" (Cardoso \& Cocco, 2003, p. 6).

Em outro estudo sobre projeto de vida de adolescentes de classe baixa, Teixeira (2005) destacou a importância da família como rede de apoio na construção do projeto de vida dos adolescentes. Essa importância se revelou quando muitos adolescentes expressaram precisar do apoio da família para a construção do projeto, além do fato de terem projetos relacionados à formação de sua própria família ou a conseguirem fornecer uma vida melhor para sua família já constituída (Teixeira, 2005).

A família é tida como a instituição que exerce maior influência na formação dos adolescentes (Sallas, 1999), dessa forma, a falta de recursos que famílias menos favorecidas enfrentam pode fazer com que o cotidiano do adolescente seja marcado por inúmeros sacrifícios em prol da família (Teixeira, 
2005). Sendo assim, é possível dizer que o projeto do adolescente pode ser completamente influenciado por suas famílias, que podem ser fonte de estímulo ou não para o jovem.

Em relação à escola, sabe-se que, depois da família, ela é o contexto no qual o adolescente passa mais tempo inserido ( $\mathrm{Li}, 2001$ ), de modo que é nesse espaço que o adolescente constrói parte de seu projeto de vida. Nesse sentido, de acordo com novas mudanças na Lei de Diretrizes e Bases da Educação Nacional (LDB), realizadas em 2017, um dos conteúdos a ser trabalhado no Ensino Médio é o projeto de vida (Lei $\left.\mathrm{n}^{\circ} 13.415,2017\right)$. Sendo assim, agora, além de ser benéfico ao adolescente, trabalhar e promover projetos de vida é um dever dos profissionais do âmbito escolar.

Apesar do estabelecimento recentemente da questão do projeto de vida no currículo escolar do Ensino Médio, já é visto que nas escolas de Programa de Ensino Integral (PEI) já se trabalha essa temática e o projeto de vida é o centro das ações de ensino. No PEI, além das disciplinas obrigatórias, os alunos cursam disciplinas eletivas, que são matérias que eles escolhem de acordo com seu objetivo (Fodra \& Nogueira, 2017). O grande diferencial desse modelo é o fato de que ele fornece condições para que o aluno elabore seu projeto de vida.

Considerando a importância do contexto escolar, diversos pesquisadores buscaram compreender a construção do projeto de vida dos adolescentes em diferentes tipos de contexto escolar (Valore \& Viaro, 2007; Zonta, 2007). Valore e Viaro (2007) buscaram compreender os elementos presentes no projeto de vida de adolescentes do ensino médio, de escola pública e particular, participantes de um projeto de orientação profissional. Verificou-se que a dimensão de projetos de vida mais citada pelos estudantes foi a profissão, porém, esse aspecto foi ainda mais presente nos discursos dos adolescentes de escola pública. Projetos de vida em relação a constituição de família correspondem ao segundo mais citado, em ambos os tipos de escola. Quanto a projetos de melhoria do mundo, apesar de ter sido a categoria menos citada, observou-se que a maioria dos adolescentes que a citaram era de escolas públicas.

Já Zonta (2007) teve como objetivo, em seu estudo, observar o modo como os adolescentes de ensino médio de escolas públicas construíam seus projetos. O estudo mostrou que, quanto aos projetos de vida, o principal objetivo apontado por eles era a realização profissional, seguido pela independência financeira. Os projetos ligados a constituição de uma família, quanto citados, foram esboçados para após o adolescente conseguir a realização profissional.

Os estudos citados fortalecem a ideia de que o projeto de vida do adolescente está diretamente vinculado ao contexto familiar e escolar e possibilitam a reflexão sobre o papel e impacto de ambos em sua construção. Uma vez que é difícil elaborar projetos de vida na atualidade, faz-se necessário incentivar e auxiliar os adolescentes nessa construção, encorajando-os a seguir suas aspirações.

Sendo assim, considerando que a construção de projeto de vida gera inúmeros benefícios para o adolescente e que projeto de vida recentemente foi incluído como um dos conteúdos a serem trabalhado no Ensino Médio, de acordo com a LDB no 13.415, de 2017, esta pesquisa buscou investigar as possíveis relações e influências das variáveis dos contextos escolar e familiar na elaboração do projeto de vida durante a adolescência. Especificamente, pretendeu-se: a) verificar a diferença entre os projetos de vida de adolescentes de diferentes sexos; b) avaliar os projetos de vida de adolescentes de diferentes anos escolares; c) verificar a diferença entre os projetos de vida de adolescentes de três tipos de escolas (escolas públicas, escolas particulares e escolas públicas do Programa de Ensino Integral); e d) analisar a relação entre o projeto de vida de adolescentes e a escolaridade materna e paterna. Partiu-se da hipótese de que os projetos de vida de adolescentes de diferentes sexos e tipos de escola teriam diferenças significativas. Além disso, esperava-se que a escolaridade parental tivesse alguma relação com projetos de vida.

\section{Método}

\section{Participantes}

Os participantes deste estudo foram 320 estudantes do Ensino Fundamental II e do Ensino Médio, de 14 a 17 anos, de ambos os sexos. Esses estudantes provinham de três diferentes tipos de escolas: escola particular $(23,7 \%, 76$ alunos), escola pública (30\%, 96 alunos) e escola pública de Programa de Ensino Integral (46,3\%, 148 alunos). 


\section{Instrumentos}

Utilizou-se a Ficha de Dados Sociodemográficos, composta por dados de identificação, configuração familiar e escolaridade. Essa ficha teve como objetivo possibilitar uma descrição da amostra e coletar informações sobre o participante e sua família.

Também utilizou-se a Escala de Projetos de Vida para Adolescentes (EPVA) (Dellazzana-Zanon, Zanon, Noronha, Oliveira, \& Rosado, 2019), uma escala Likert de cinco pontos, composta por 48 itens. Os itens da EPVA são englobados em cinco dimensões, a saber: a) Relacionamentos Afetivos; b) Estudo; c) Aspirações Positivas; d) Bens Materiais; ee) Religião/Espiritualidade.

\section{Procedimentos de coleta de dados}

Após a aprovação do projeto de pesquisa pelo Comitê de Ética em Pesquisa com Seres Humanos da Pontifícia Universidade Católica de Campinas (CAAE 88816418.5.0000.5481), entrou-se em contato com as escolas para obtenção de suas autorizações para a realização da coleta de dados. Foi agendado um dia para que a pesquisadora fosse até a escola para explicar a pesquisa aos alunos e entregar o Termo de Consentimento Livre e Esclarecido (TCLE) para que eles o levassem para casa e seus responsáveis legais pudessem consentir sua participação na pesquisa. Além disso, foram agendados dia e horário para realização da coleta de dados. No dia da aplicação, os alunos que trouxeram o Termo de Consentimento Livre e Esclarecido assinado pelos pais também assinaram um Termo de Assentimento Livre e Esclarecido (TALE). Em seguida, foram esclarecidos eventuais questionamentos e entregues os dois instrumentos da pesquisa: a) Ficha de Dados Sociodemográficos; e b) Escala de Projeto de Vida para Adolescentes. A pesquisadora leu as instruções dos instrumentos com os alunos e explicou como preencher de modo adequado cada um deles. A aplicação durou 50 minutos.

\section{Resultados}

\section{Caracterização dos participantes}

Em relação ao sexo dos participantes, a amostra do estudo contou com 320 adolescentes, dos quais 203 $(63,4 \%)$ eram do sexo feminino e $117(36,6 \%)$, do sexo masculino. Quanto à escolaridade das mães e dos pais dos participantes, segue a Tabela 1:

Tabela 1

Nível de Escolaridade das Mães e dos Pais dos Participantes

\begin{tabular}{ccccccc}
\hline \multirow{2}{*}{ Escolaridade } & \multicolumn{2}{c}{ Escola integral } & \multicolumn{2}{c}{ Escola particular } & \multicolumn{2}{c}{ Escola pública } \\
\cline { 2 - 6 } & Mães & Pais & Mães & Pais & Mães & Pais \\
\hline Ensino Fundamental Incompleto & $18,3 \%$ & $21,9 \%$ & $2,7 \%$ & $2,85 \%$ & $19,0 \%$ & $24,65 \%$ \\
Ensino Fundamental Completo & $6,8 \%$ & $7,0 \%$ & $6,8 \%$ & $5,71 \%$ & $17,8 \%$ & $9,58 \%$ \\
Ensino Médio Incompleto & $9,5 \%$ & $9,2 \%$ & $2,7 \%$ & $5,71 \%$ & $21,4 \%$ & $13,69 \%$ \\
Ensino Médio Completo & $40,1 \%$ & $37,5 \%$ & $26,0 \%$ & $28,57 \%$ & $23,8 \%$ & $30,13 \%$ \\
Ensino Superior Incompleto & $6,1 \%$ & $4,2 \%$ & $2,7 \%$ & $2,85 \%$ & $1,1 \%$ & $2,73 \%$ \\
Ensino Superior Completo & $12,2 \%$ & $12,0 \%$ & $28,7 \%$ & $30 \%$ & $9,5 \%$ & $10,95 \%$ \\
Pós-Graduação & $6,8 \%$ & $7,8 \%$ & $30,1 \%$ & $24,28 \%$ & $7,1 \%$ & $8,21 \%$ \\
\hline
\end{tabular}

\section{Projeto de vida e variáveis do contexto escolar e familiar}

\footnotetext{
Projeto de vida e sexo

$\mathrm{O}$ teste $t$ de student para comparar os projetos de vida dos adolescentes (Tabela 2) de acordo com o sexo, indicou que existe uma diferença significativa entre os projetos de vida dos participantes do sexo masculino e do sexo feminino nas dimensões
}

Aspirações Positivas e Estudo. Na dimensão Aspirações Positivas, as participantes do sexo feminino apresentaram uma média superior $(\mathrm{M}=4,54$; $D P=0,43)$ à do sexo masculino $(\mathrm{M}=4,33 ; D P=0,51)$. O mesmo ocorreu na dimensão Estudo, na qual as estudantes também obtiveram um resultado superior $(\mathrm{M}=4,44$; $\mathrm{DP}=0,41)$ em relação ao dos estudantes $(\mathrm{M}=4,27 ; \mathrm{DP}=0,68)$. Quanto às outras dimensões não houve diferença significativa. 
Tabela 2

Teste $t$ de student entre projetos de vida e sexo.

\begin{tabular}{|c|c|c|c|c|c|c|}
\hline Dimensões de PV & Sexo & $M$ & $D P$ & $t$ & $g l$ & $p<$ \\
\hline \multirow{2}{*}{ Religião/Espiritualidade } & Feminino & 3,85 & 0,92 & \multirow{2}{*}{1} & \multirow{2}{*}{199} & \multirow{2}{*}{0,30} \\
\hline & Masculino & 3,72 & 1,15 & & & \\
\hline \multirow{2}{*}{ Aspirações Positivas } & Feminino & 4,54 & 0,44 & \multirow{2}{*}{3,6} & \multirow{2}{*}{314} & \multirow{2}{*}{0,001} \\
\hline & Masculino & 4,34 & 0,52 & & & \\
\hline \multirow{2}{*}{ Bens Materiais } & Feminino & 4,54 & 0,42 & \multirow{2}{*}{$-0,1$} & \multirow{2}{*}{314} & \multirow{2}{*}{0,91} \\
\hline & Masculino & 4,34 & 0,48 & & & \\
\hline \multirow{2}{*}{ Estudo } & Feminino & 4,45 & 0,42 & \multirow{2}{*}{2,4} & \multirow{2}{*}{160} & \multirow{2}{*}{0,01} \\
\hline & Masculino & 4,27 & 0,69 & & & \\
\hline \multirow{2}{*}{ Relacionamento afetivo } & Feminino & 3,70 & 0,72 & \multirow{2}{*}{0,3} & \multirow{2}{*}{314} & \multirow{2}{*}{0,7} \\
\hline & Masculino & 3,67 & 0,83 & & & \\
\hline
\end{tabular}

PV=projeto de vida, $M=$ média, $D P=$ desvio-padrão, $t=$ estatística $t, g l=$ graus de liberdade, $p=$ probabilidade usada no teste da hipótese nula que ajuda a rejeitá-la ou não rejeitá-la.

\section{Projeto de vida e séries escolares}

Foi realizada uma análise de variância (ANOVA) one-way para verificar as possíveis diferenças entre os Projetos de Vida dos adolescentes de diferentes séries escolares ( $9^{\circ}$ ano do Ensino Fundamental, $1^{\circ}$ ano do Ensino Médio, $2^{\circ}$ ano do Ensino Médio e $3^{\circ}$ ano do Ensino Médio). Os resultados mostraram que houve diferença significativa quanto às séries escolares e às dimensões: a) Religião/Espiritualidade [F $(3,313)=3,54 ; \quad \mathrm{p}<0,015] ;$ b) Relacionamentos Afetivos [F $(3,311)=2,525 ; \mathrm{p}<0,05]$; e c) Bens Materiais $[\mathrm{F}(3,311)=3,276 ; \mathrm{p}<0,021]$.

Para a dimensão Religião/Espiritualidade, testes post hoc (Tuckey) indicaram diferenças significativas entre: a) o nono ano $(\mathrm{M}=4,05 ; \mathrm{DP}=0,91)$ e o $1^{\circ}$ ano do Ensino Médio ( $\mathrm{M}=3,67$; $\mathrm{DP}=1,11)$; b) o nono ano e $3^{\circ}$ ano do Ensino Médio ( $\mathrm{M}=3,56$; $\mathrm{DP}=1,03$ ). Esses resultados indicam que conforme os adolescentes avançam nos anos escolares, há uma tendência de que os projetos de vida referentes a essa dimensão diminuam. Ou seja, os adolescentes mais novos ( $9^{\circ}$ ano do Ensino Fundamental) apresentaram mais projetos de vida nessa dimensão do que adolescentes mais velhos.

No que diz respeito à dimensão Relacionamentos Afetivos, testes posthoc (Tuckey) indicaram diferençassignificativas entre $01^{\circ}$ ano do Ensino Médio (M=3,57;DP=0,76) eo3 ${ }^{\circ}$ ano doEnsino Médio ( $\left.\mathrm{M}=3,9 ; \mathrm{DP}=0,70\right)$. Observou-se que os adolescentes mais velhos ( $3^{\circ}$ ano do Ensino Médio) têm mais projetos de vida nessa dimensão do que os mais novos ( $1^{\circ}$ ano do Ensino Médio), ou seja, existe uma tendência de os projetos de Relacionamentos Afetivo aumentarem com a escolaridade.

Quanto à dimensão Bens Materiais, testes post hoc (Tuckey) indicaram diferenças significativas entre o $9^{\circ}$ ano do Ensino Fundamental $(\mathrm{M}=4,6 ; \mathrm{DP}=0,42)$ e o $3^{\circ}$ ano do Ensino Médio $(M=4,3$; $D P=0,45)$. Sendo assim, é possível observar que os adolescentes mais novos ( $9^{\circ}$ ano do Ensino Fundamental) têm mais projetos de vida nesse aspecto do que os mais velhos ( $3^{\circ}$ ano do Ensino Médio). Além disso, os dados mostraram que existe uma tendência de que os projetos de vida quanto a Bens Materiais diminuam à medida que os adolescentes crescem. Quanto às demais dimensões (Aspirações Positivas e Estudo), não foram observadas diferenças significativas entre os projetos de vida nos diferentes anos escolares.

\section{Projetos de vida e tipos de escolas}

Foi realizada uma análise de variância (ANOVA) para avaliar possíveis diferenças entre os projetos de vida dos adolescentes de diferentes tipos de escola: a) escola particular; b) escola pública; ec) escola pública de Programa de Ensino Integral. Os resultados mostraram diferenças significativas quanto aos tipos de escola e as dimensões: a) Religião e Espiritualidade $[\mathrm{F}(2,315)=16,00$; $\mathrm{p}<001]$; b) Bens Materiais [F $(2,313)=5,13 ; \mathrm{p}<006]$; e c) Relacionamentos Afetivos $[\mathrm{F}(2,313)=3,62 ; \mathrm{p}<028]$. 
Quanto aos projetos de vida da dimensão Religião/ Espiritualidade, testes post hoc (Tuckey) indicaram que: a) escola particular $(\mathrm{M}=3,2$; $\mathrm{DP}=1,2)$ apresentou escores inferiores a escolas públicas $(\mathrm{M}=3,9$; $\mathrm{DP}=0,84)$; e b) escola particular apresentou escores inferiores a escola pública de Programa de Ensino Integral $(\mathrm{M}=4,0 ; \mathrm{DP}=0,8)$. Esses resultados mostram que alunos de escola particular apresentam níveis inferiores de projetos de vida relacionados à Religião/Espiritualidade que alunos de outros tipos de escolas (Figura 1).

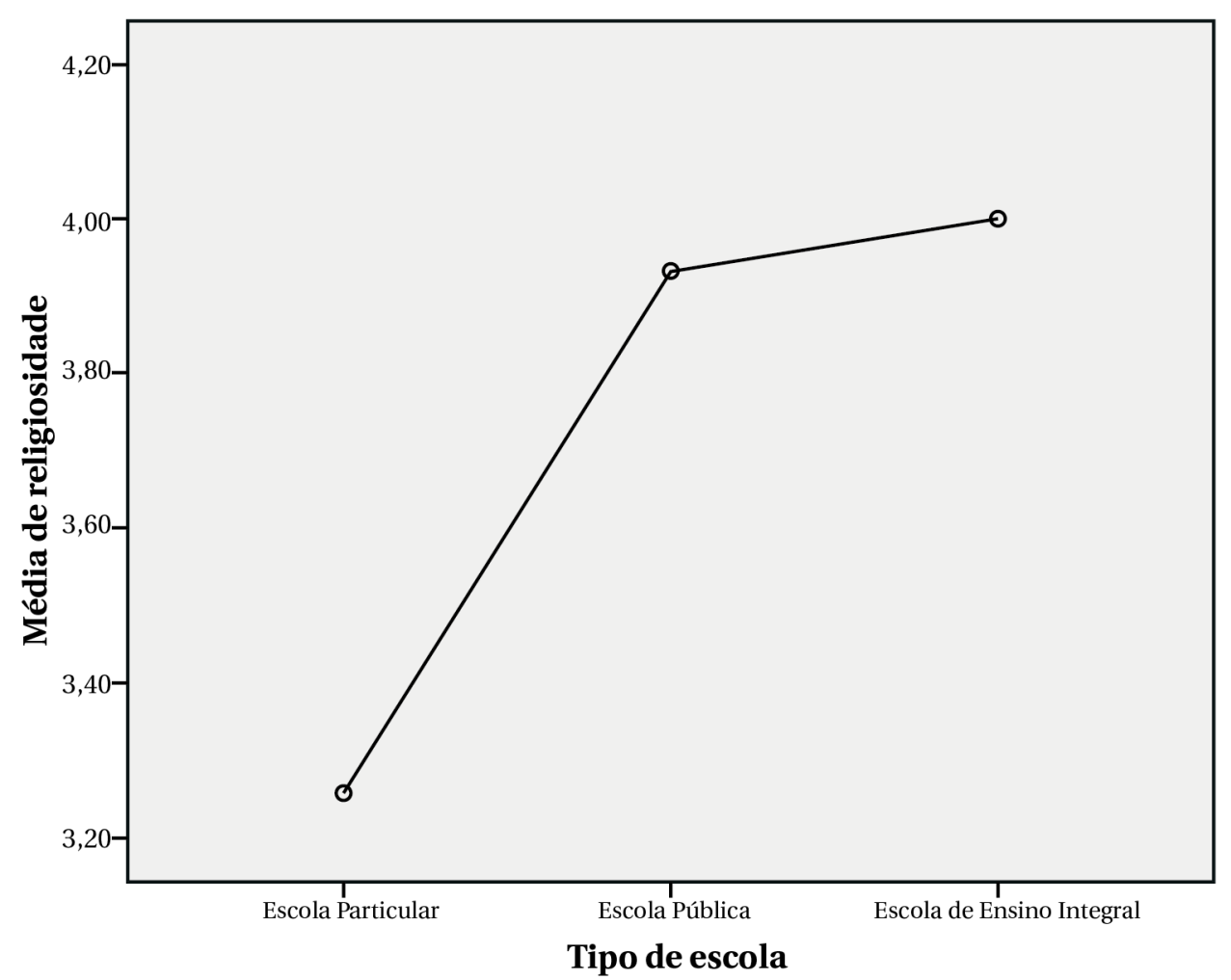

Figura 1

Relação entre projetos de vida de Religião/Espiritualidade com o tipo de escola.

Outra dimensão que apresentou diferenças significativas nos projetos de vida dos participantes foi a Bens Materiais. Em relação aos projetos de vida dessa dimensão, testes post hoc (Tuckey) indicaram que: a) escola pública de Programa de Ensino Integral $(\mathrm{M}=4,5 ; \mathrm{DP}=0,41)$ apresentou escores superiores aos da Escola Particular $(\mathrm{M}=4,4 ; \mathrm{DP}=0,46)$; e b) escola pública de Programa de Ensino Integral apresentou escores superiores aos da escola pública $(\mathrm{M}=4,4 ; \mathrm{DP}=0,45)$ (Figura 2).

Já nos projetos de vida da dimensão Relacionamentos Afetivos, testes post hoc (Tuckey) também indicaram diferenças. As análises mostraram que escola particular $(\mathrm{M}=3,5 ; \mathrm{DP}=0,80)$ tem escores inferiores aos da escola pública ( $\mathrm{M}=3,8 ; \mathrm{DP}=0,63)$. Ou seja, adolescentes da escola pública têm mais projetos de vida na dimensão Relacionamentos Afetivos do que os estudantes da escola particular. Quanto aos projetos de vida da escola pública e da escola pública de Programa de Ensino Integral, não foram encontradas diferenças significativas. O mesmo ocorreu quando os projetos de vida dos adolescentes da escola pública de Programa de Ensino Integral foram comparados aos da escola particular. Ou seja, a diferença significativa nessa dimensão se manifestou apenas quando comparados os projetos entre a escola pública e a particular (Figura 3). 
Pereira, B. C., Zanon, C., \& Dellazzana-Zanon, L. L. (2021). Influência de contextos no projeto de vida.

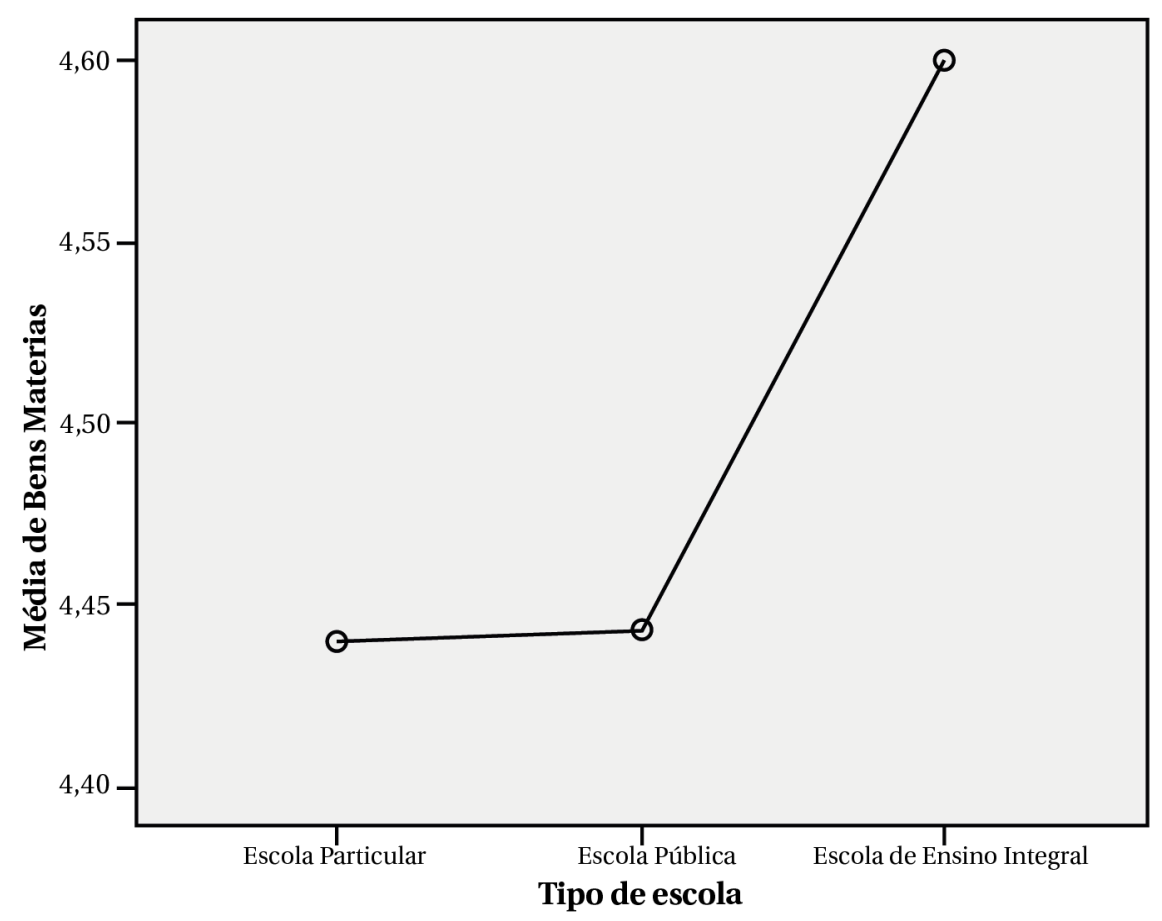

Figura 2

Relação entre projeto de vida da dimensão bens materiais com o tipo de escola.

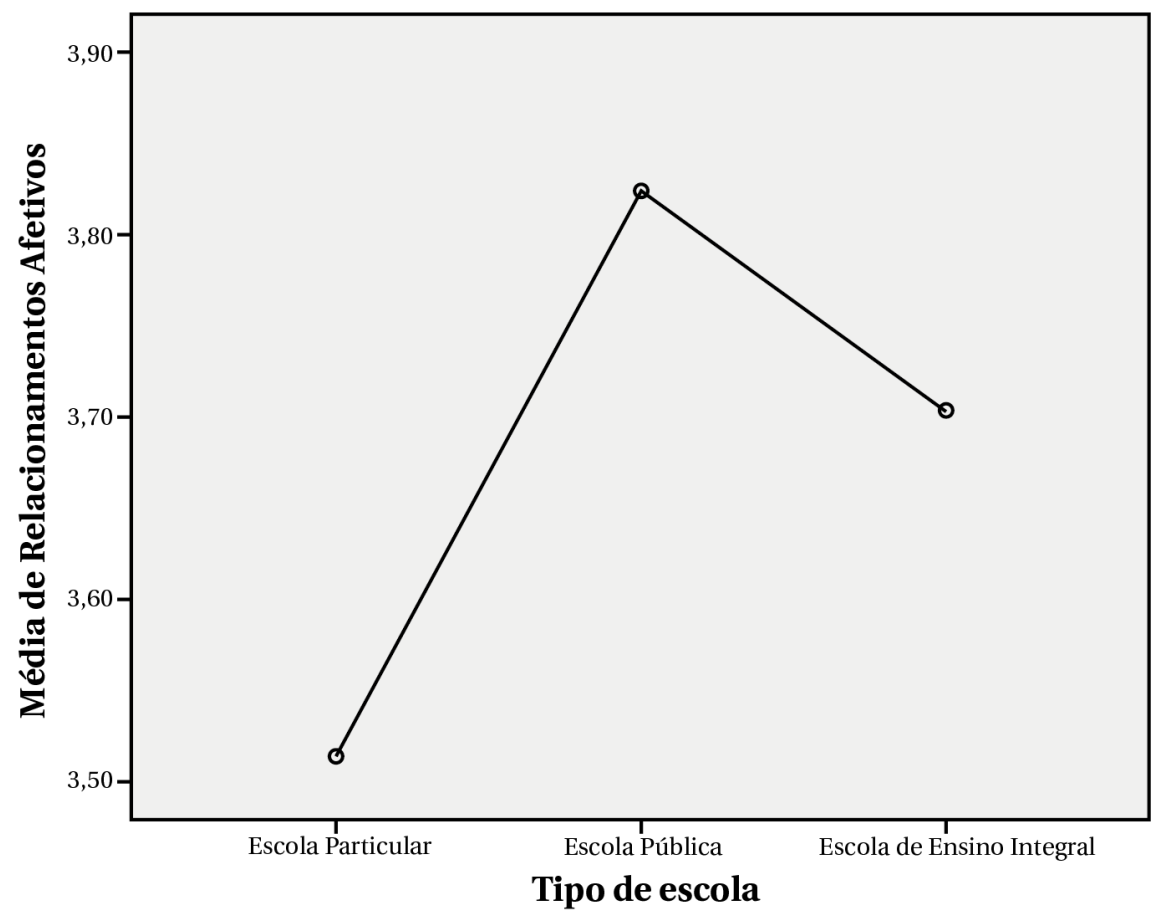

Figura 3

Relação entre projeto de vida da dimensão Relacionamentos Afetivos e o tipo de escola. 


\section{Projeto de vida e escolaridade parenta}

Por meio da correlação de Pearson, foram verificadas as possíveis correlações existentes entre: a) as dimensões da EPVA (Religião/Espiritualidade, Aspirações Positivas, Bens Materiais, Estudo, Relacionamentos
Afetivos); b) Escolaridade da Mãe e as dimensões da EPVA; c) Escolaridade do Pai e as dimensões da EPVA; d) Repetição de Ano Escolar e as dimensões da EPVA; e) Abandono Escolar e as dimensões da EPVA; ef) Expulsão da Escola e as dimensões da EPVA (Tabela3).

Tabela 3

Correlação entre as dimensões da EPVA e as variáveis do contexto escolar e familiar.

\begin{tabular}{lcccccccccc}
\hline \multicolumn{1}{c}{ Dimensões } & $(1)$ & $(2)$ & $(3)$ & $(4)$ & $(5)$ & $(6)$ & (7) & (8) & (9) & (10) \\
\hline (1) Religião/Espiritualidade & - &. &. &. &. &. &. &. &. & - \\
(2) Aspirações Positivas & $0,32^{* *}$ & - &. &. &. &. &. &. &. &. \\
(3) Bens Materiais & $0,23^{* *}$ & $0,37^{* *}$ & - &. &. &. &. &. &. &. \\
(4) Estudo & $0,12^{*}$ & $0,33^{* *}$ & 0,10 & - &. &. &. &. &. &. \\
(5) Relacionamento Afetivo & $0,25^{* *}$ & $0,19^{* *}$ & $0,28^{* *}$ & 0,02 & - &. &. &. &. &. \\
(6) Escolaridade da Mãe & $-0,13^{*}$ & 0,04 & $-0,02$ & 0,02 & $-0,01$ & - &. &. &. &. \\
(7) Escolaridade do Pai & $-0,12^{*}$ & 0,04 & $-0,02$ & 0,01 & 0,01 & $0,52^{* *}$ & - &. &. &. \\
(8) Repetiu algum ano & $-0,01$ & $-0,07$ & $-0,02$ & $-0,12^{*}$ & $-0,10$ & $-0,02$ & $-0,04$ & - &. &. \\
(9) Abandonou a escola & 0,01 & $-0,04$ & $-0,01$ & $-0,02$ & 0,01 & $-0,07$ & $-0,02$ & $0,30^{* *}$ & - &. \\
(10) Expulso da escola & $-0,001$ & $-0,01$ & $-0,01$ & 0,01 & 0,01 & $-0,03$ & 0,04 & $0,14^{*}$ & $0,21^{* *}$ & - \\
\hline
\end{tabular}

Quanto à relação entre a escolaridade da mãe e do pai e as dimensões da EPVA, os resultados revelaram que houve correlação negativa entre: a) escolaridade da mãe e a dimensão Religião/Espiritualidade; e b) escolaridade do pai e a dimensão Religião/ Espiritualidade. Isso significa que conforme a escolaridade do pai ou da mãe aumenta, menos são os projetos referentes a Religião/Espiritualidade e vice-versa. Para as demais dimensões, não foi constatada correlação entre a escolaridade da mãe e os projetos.

\section{Discussão}

Foi possível avaliar os projetos de vida de adolescentes de diferentes sexos e idades e também verificar se existiam diferenças entre eles em relação às variáveis dos contextos escolar e familiar. Ao analisar os projetos de vida dos adolescentes de diferentes sexos (feminino e masculino), pode-se perceber que, em algumas dimensões, as participantes do sexo feminino tinham mais projetos de vida do que os participantes do sexo masculino. Elas apresentaram mais projetos nas dimensões Estudo e Aspirações Positivas. Considerando que as adolescentes tinham muitos projetos de vida em relação a essas duas dimensões, é possível concluir que realizar uma faculdade ou curso técnico e se formar é uma prioridade mais imediata para as participantes do sexo feminino do que os outros aspectos do projeto de vida. Em contrapartida, os adolescentes do sexo masculino, apesar de terem projetos de vida nessas dimensões, não apresentam tantos quanto as participantes do sexo feminino.

Em relação ao contexto escolar, as análises dos projetos de vida dos adolescentes de diferentes anos revelaram que os adolescentes mais novos $\left(9^{\circ}\right.$ ano do Ensino Fundamental) têm mais projetos de vida do que os mais velhos (Ensino Médio) nas dimensões Religião/Espiritualidade e Bens Materiais.

Em Bens Materiais, foi possível observar que conforme os adolescentes crescem, diminuem os projetos de vida dessa dimensão, pois os mais novos se preocupam mais em adquirir itens como roupas de marcas famosas, veículos e fazer viagens, por exemplo, do que os mais velhos. Isso pode ocorrer devido a esses adolescentes mais novos terem um pensamento mais imediatista, cuja lógica é a de satisfazer os desejos e necessidades momentâneos, sobretudo quando se considera a sociedade em que estamos inseridos, na qual o adolescente é induzido a associar status e poder a aquisições materiais (Serrão \& Beleeiro, 1999). Sendo assim, é possível concluir que os adolescentes 
mais velhos têm menos projetos de vida nessa dimensão por terem projetos mais voltados a necessidades básicas e não tão supérfluas.

Além disso, foi visto que os adolescentes mais velhos apresentaram outras prioridades: os Relacionamentos Afetivos. Tal fato se confirmou nos resultados da pesquisa, que mostraram que os adolescentes mais velhos ( $3^{\circ}$ ano do Ensino Médio) possuem mais projetos de vida nessa dimensão do que os adolescentes mais novos (9o ano do Ensino Fundamental). É visto que os relacionamentos na adolescência são marcados pela inexistência de compromisso. $\mathrm{O}$ ato de "ficar", que é um tipo de relacionamento afetivo muito praticado pelos adolescentes, tem por características ser breve, imediatista e descompromissado (Justo, 2005).

Nessa fase do desenvolvimento, relacionamentos sem compromissos são aceitos socialmente e até valorizados entre os adolescentes. Porém, ainda que esses relacionamentos sejam muito praticados pelos adolescentes, estes relatam, como planos para o futuro, projetos de casar e constituir uma família (Sousa, Nunes, \& Machado, 2012). Ou seja, eles atribuem os relacionamentos mais superficiais à adolescência e não os veem como algo que pretendam fazer durante a vida adulta (Sousa, et al., 2012). É possível dizer que os adolescentes mais velhos têm mais projetos de vida na dimensão Relacionamentos Afetivos do que os mais novos porque ingressarão na vida adulta mais brevemente, ou seja, a ideia de ter um relacionamento mais sério está mais próxima da realidade deles do que dos mais novos.

Em relação à dimensão Religião/Espiritualidade, os adolescentes mais novos ( $9^{\circ}$ ano do Ensino Fundamental) têm mais projetos do que os adolescentes mais velhos ( $3^{\circ}$ ano do Ensino Médio). A adolescência pode ser tida como uma fase na qual ocorre um despertar religioso, em que os fenômenos religiosos aparecem de modo intenso nos sentimentos e pensamentos do adolescente (Camboim \& Rique, 2010). Devido a esses sentimentos intensos, alguns adolescentes apresentam um posicionamento radical, seja em forma de crença fervorosa seja de um ateísmo extremo (Camboim \& Rique, 2010), o que pode ter se refletido nos resultados dos adolescentes mais novos.

Em estudo realizado por Camboim e Rique (2010), que buscava verificar a diferença entre a religiosidade de jovens adultos e de adolescentes, foi constatado que os jovens adultos, apesar de apresentarem maior espiritualidade, praticavam menos sua religião e eram menos religiosos que os adolescentes. Esse resultado está em consonância com os deste estudo, no qual foi verificada uma tendência de os projetos de vida da dimensão Religião/Espiritualidade diminuírem à medida que os adolescentes crescem.

Ainda quanto aos aspectos do contexto escolar, foram analisadas as diferenças entre os projetos de vida dos diferentes tipos de escolas, sendo elas: a) escola particular; b) escola pública; e c) escola pública do Programa Ensino Integral. Observou-se que houve diferença entre os projetos de vida nas dimensões Religião/Espiritualidade, Relacionamentos Afetivos e Bens Materiais. Por meio das análises, constatou-se que os adolescentes de escola pública apresentaram mais projetos de vida na dimensão Relacionamentos Afetivos do que os estudantes dos demais tipos de escola (particular e de Ensino Integral). Isso pode significar que os adolescentes de escolas públicas têm: a) projetos de vida de constituir uma família mais cedo do que os adolescentes dos outros tipos de escola, o que justificaria o fato de esses projetos aparecerem nesse momento, esboçados para os próximos 10 anos; e/ou b) que para esses adolescentes, a questão dos relacionamentos afetivos tem mais relevância do que para os demais.

De acordo com Bauman (1998), a contemporaneidade fez com que os relacionamentos afetivos, que antes eram projetos que orientavam o ser humano para objetivos que iam além do próprio prazer sexual, se desconectassem de outras dimensões de projeto de vida. Ou seja, eles seriam voltados apenas à função da busca de prazer. Mas, ao contrário da afirmação de Bauman, estudos recentes mostram que, para muitos adolescentes, a construção de projetos de vida na dimensão Relacionamentos Afetivos está diretamente atrelada a outros fatores como a estabilidade financeira, que seria alcançada por meio de um bom emprego após a conclusão de um curso técnico ou universitário (DellazzanaZanon, 2014; Valore \& Viaro, 2007).

Pode-se levantar a hipótese de que os adolescentes das escolas particulares e escolas públicas de Ensino Integral não apresentam tantos projetos em relação à dimensão Relacionamentos Afetivos por estarem mais preocupados em primeiramente se formar, ingressar no mercado de trabalho e conquistar a independência financeira. Nesse sentido, os resultados de um estudo que investigou os projetos de vida sobre relacionamentos afetivos de adolescentes de nível socioeconômico baixo mostraram 
que devido, à urgência de concretizar projetos quanto ao trabalho e ao estudo, projetos ligados ao casamento e a ter filhos (Relacionamentos Afetivos) tendem a ser colocados em segundo plano (Riter, Dellazzana-Zanon, \& Freitas, 2019).

É interessante observar que os estudantes da escola pública de Ensino Integral apresentaram mais projetos de vida relacionados a Bens Materiais do que os estudantes de escolas públicas e escolas particulares. Nota-se que os estudantes da escola pública de Ensino Integral desejam e buscam a independência financeira e a conquista de bens, o que poderia estar relacionado com o fato de não terem tantos projetos de vida na dimensão Relacionamentos Afetivos, pois buscam estabilidade e boa qualidade de vida antes de constituírem uma família. Essas duas dimensões de projeto de vida estão correlacionadas, como indicaram os resultados desta pesquisa, na qual se constata uma tendência de que quanto mais projetos de vida em relação a Bens Materiais, menos serão os projetos de vida em relação a Relacionamentos Afetivos. Talvez isso aconteça justamente devido a essa priorização.

Além disso, é possível pensar que esse resultado está relacionado à diferença socioeconômica entre os adolescentes dos diferentes tipos de escola. Para os estudantes de escolas particulares, devido a sua condição financeira, os projetos da dimensão Bens Materiais não são tão necessários; o que não ocorre com os estudantes dos outros tipos de escola, que não têm tal condição. No Brasil, o tipo de escola é um marcador de diferenças de nível socioeconômico na medida em que as diferenças de classe social são reproduzidas há muitos anos no sistema educacional no Brasil (Guzzo \& Euzébios Filho, 2005). Dito de outra forma, os projetos de vida dos adolescentes, principalmente quanto à dimensão Bens Materiais, são influenciados pela condição socioeconômica dos adolescentes. A região metropolitana de Campinas, local onde foram realizadas as coletas, apresenta grande desigualdade social e alto nível de segregação social (Cunha \& Falcão, 2017), o que pode ter refletido nas diferenças entre os projetos de vida de escolas diferentes.

Em relação à dimensão Religião/Espiritualidade, foi observado que os projetos de vida dos adolescentes da escola particular são menores do que os dos adolescentes dos outros dois tipos de escola. Ou seja, é possível analisar que os estudantes de escola particular não atribuem tanta relevância a Religião/ Espiritualidade na construção de seu projeto de vida. Para analisar tal dado, cabe aqui discutir outro dado relevante que foi apresentado nos resultados referentes à influência do contexto familiar nos projetos de vida do adolescente: escolaridade da mãe e escolaridade do pai. Os dados mostraram a existência de uma correlação negativa entre: a) escolaridade da mãe e a dimensão Religião/Espiritualidade; e b) escolaridade do pai e Religião/Espiritualidade. Ou seja, à medida que aumenta a escolaridade parental, diminuem os projetos de vida dos filhos em relação à dimensão Religião/Espiritualidade.

Desse modo, nota-se uma consonância entre os resultados da comparação da dimensão Religião / Espiritualidade ao tipo de escola e às escolaridades parentais, uma vez que os pais com maior escolaridade são os dos participantes que estudam na escola particular. É importante ressaltar que foi encontrada uma correlação positiva entre as dimensões de projetos de vida Religião/Espiritualidade e Aspirações Positivas. Os projetos da dimensão Aspirações Positivas são aqueles voltados ao desejo de se melhorar enquanto pessoa ao longo do tempo, como projetos que indiquem a vontade de se tornar alguém melhor e que faça diferença na sociedade e na vida de outras pessoas (Pereira, Gobbo, \& Dellazzana-Zanon, 2017). Esse é um fato interessante na medida em que suscita a reflexão sobre os motivos dessa correlação positiva, de modo que é possível levantar algumas hipóteses sobre isso.

Em um estudo realizado por Valore e Viaro (2007), que investigou os projetos de vida de adolescentes do Ensino Médio em relação à profissão e à sociedade, partiu-se da hipótese de que os projetos teriam predominantemente um caráter individualista, devido às características de nossa sociedade. Entretanto, ao contrário do esperado, os resultados do estudo mostraram que, em relação à contribuição social do exercício profissional, $79,8 \%$ dos participantes conseguiram pensar em alguma ação voltada ao coletivo. Um fato que chamou atenção nesse estudo é de que entre os 8 participantes que demonstraram projetos de contribuir com a população carente por meio do exercício profissional, 7 deles eram de escolas públicas. Ou seja, os estudantes de escolas públicas mostraram uma preocupação maior com o outro e com a realidade social na qual eles estão inseridos, do que os estudantes de escolas particulares, que manifestaram projetos mais individualistas. Esses projetos de vida dos participantes do estudo de 
Valore e Viaro (2007) poderiam ser enquadrados na dimensão Aspirações Positivas.

Considerando os resultados deste estudo e os do estudo de Valore e Viaro (2007), poder-se-ia levantar a hipótese de que os participantes da escola particular apresentaram menos projetos de vida em relação à Religião/Espiritualidade por terem projetos de vida mais individualistas, uma vez que tais dimensões estão correlacionadas. Sendo assim, conclui-se que o contexto escolar pode influenciar na maneira como o indivíduo se relaciona com o mundo a sua volta e que, também, as escolas particulares podem estar, de certa forma, contribuindo para que seus estudantes tenham uma subjetividade mais individualista (Valore \& Viaro. 2007).

Quanto à dimensão Estudo, era esperado que a escolaridade parental estivesse principalmente correlacionada positivamente a ela, uma vez que se sabe que existe uma associação entre resultados escolares positivos e uma maior escolaridade dos pais (Banco Mundial \& Unicef, 1997). Além disso, estudos ressaltam que sobretudo o nível de escolaridade da mãe é determinante para o desempenho escolar de seu filho (Banco Mundial \& Unicef, 1997). Isso ocorre porque mães com maior escolaridade têm mais envolvimento com a escolarização dos filhos, o que acarreta um melhor desempenho deles nos estudos (Stevenson \& Baker, 1987). Apesar disso, ao contrário do que se esperava e indicava a literatura, não foram encontradas correlações entre escolaridade da mãe e a dimensão Estudo.

Também sobre a dimensão Estudo, não foram averiguadas diferenças entre os projetos de vida dos adolescentes de diferentes tipos de escola, conforme previsto na hipótese inicial deste estudo. Em uma pesquisa realizada por Sarriera, Silva, Kabbas e Lópes (2001) com adolescentes de escolas públicas, os resultados indicaram que os projetos de vida dos adolescentes desse tipo de escola eram pouco definidos e que havia pouco interesse deles em projetos na dimensão Estudo. Sendo assim, esperava-se que os adolescentes da escola pública de Ensino Integral e da escola particular tivessem mais projetos de vida na dimensão Estudo do que os da escola pública, o que não ocorreu. Em relação aos projetos de vida dos estudantes da escola pública de Ensino Integral, era esperado que, especialmente nesse tipo de escola, houvesse diferenças em todas as dimensões de projetos de vida, uma vez que o projeto de vida tem papel central em sua base curricular.
Considerando, então, que nas escolas públicas de Ensino Integral os adolescentes dispõem de espaço para discutir e pensar o projeto de vida e, além disso, em princípio, recebem um ensino de qualidade e vinculado aos projetos de vida, ou seja, um ensino que faz sentido para os adolescentes, era esperado que esses fatores refletissem nos resultados deste estudo.

\section{Considerações finais}

O presente estudo teve como objetivo investigar as possíveis relações existentes entre os projetos de vida de adolescentes e os contextos sociais mais importantes e nos quais eles passam mais tempo durante essa fase do desenvolvimento: a família e a escola. Foi possível concluir que os objetivos propostos pela pesquisa foram alcançados. Por meio das análises, identificou-se quais áreas do projeto de vida têm mais relações com a família e a escola. Além disso, pode-se compreender melhor as diferenças e semelhanças entre os projetos de vida de adolescentes de idades, sexo e anos escolares diferentes.

Os resultados mostraram que adolescentes mais novos têm mais projetos de vida em relação a Relacionamentos Afetivos, Religião/Espiritualidade e Bens Materiais do que os mais velhos. Além disso, foi visto uma tendência de os projetos da dimensão Religião/Espiritualidade diminuírem conforme os adolescentes crescem, assim como uma correlação negativa com a escolaridade da mãe e a escolaridade do pai. Em relação ao tipo de escola, os resultados mostraram que os adolescentes de escolas públicas têm mais projetos de vida na dimensão Relacionamentos Afetivos que os adolescentes dos demais tipos de escola e que os projetos da dimensão Religião/Espiritualidade são maiores entre os adolescentes de escolas públicas.

Ao contrário do esperado, não foram constatadas diferenças relacionadas aos projetos da dimensão Estudo em relação ao tipo de escola. Esperava-se que os alunos da escola pública do PEI tivessem mais projetos de vida que os estudantes dos outros tipos de escola, uma vez que eles têm como um dos objetivos acadêmicos a construção de um projeto de vida. Porém, é importante citar que esse modelo de ensino ainda é muito recente. Sugere-se futuramente repetir essa coleta de dados com uma turma que tenha estudado todos os anos de sua formação na Educação Básica nesse modelo de ensino. 
Cabe ressaltar aqui que o presente estudo tem limitações. Por se tratar de uma coleta de dados, os estudantes tiveram que responder dois instrumentos e um deles, a Escala de Projeto de Vida para Adolescentes, é um instrumento longo. Dessa forma, é possível que os participantes tenham respondido sem muita atenção aos itens. Outro aspecto é o número de participantes dos diferentes tipos de escola. Houve menos estudantes da escola particular, devido à dificuldade em conseguir autorizações das instituições para a realização do estudo nesse tipo de escola. Sugere-se que, no futuro, o estudo seja repetido com números semelhantes de participantes em cada tipo de escola.

Por fim, é possível dizer que analisar a relação entre projeto de vida e as variáveis dos contextos escolar e familiar foi importante para compreender a influência que tais aspectos exercem na construção de projetos de vida do adolescente. Espera-se que essa compreensão permita que futuras intervenções sejam realizadas em escolas a fim de capacitar tanto professores quanto pais e responsáveis a trabalharem o projeto de vida com seus alunos ou filhos de forma mais específica.

\section{Referências}

Bauman, Z. (1998). O mal-estar da pós-modernidade. Zahar.

Banco Mundial \& Unicef. (1997). Chamada à ação: combatendo o fracasso escolar no Nordeste (2a ed.).

Camboim, A., \& Rique, J. (2010). Religião e espiritualidade de adolescentes e jovens adultos. Revista Brasileira de História das Religiões, 3(7), 1-13.

Cardoso, C. P., \& Cocco, M. I. M. (2003). Projeto de vida de um grupo de adolescentes à luz de Paulo Freire. Revista Latino-Americana de Enfermagem, 11(6), 778-785. https:// doi.org/10.1590/S0104-11692003000600012

Cunha, J. M. P., \& Falcão, C. A. (2017). Campinas metropolitana: Diversidades socioespaciais na virada para o século $X X I$. Librum Editora.

Damon, W. (1995). Greater Expectations: Overcoming the Culture of Indulgence in our Homes and Schools. Free Press.

Damon, W., Menon, J., \& Bronk, K. C. (2003). The Development of Purpose During Adolescence. Applied Developmental Science, 7(3), 119-128.

Dellazzana-Zanon, L. L. (2014). Projetos de vida na adolescência: Comparação entre adolescentes que cuidam e que não cuidam de seus irmãos menores [Tese de doutorado, Universidade Federal do Rio Grande do Sul].

Dellazzana-Zanon, L.L., Riter, H. S., \& Freitas, L. B. L. F. (2015). Projetos de vida de adolescentes que cuidam e que não cuidam de seus irmãos menores. In R. M. S. de Macedo (Ed.), Expandindo horizontes da terapia familiar (pp.111-122). CRV.

Dellazzana-Zanon, L. L., Zanon, C., Noronha, A. P. P., Oliveira, M.V., \& Rosado, A. F. P. (2019). Evidências Preliminares de Validade da Escala de Projetos de Vida Para Adolescentes.Avaliação Psicológica, 18(4), 429-437. https://doi.org/ 10.15689/ap.2019.1804.18602.11

Erikson, E. (1976). Identidade, juventude e crise. Zahar. (Obriga original publicada em 1968)

Fodra, S. M., \& Nogueira, M. E. C. (2017). O projeto de vida nas escolas do Programa Ensino Integral. Revista @mbienteeducação, 10(2), $251-261$.

Guzzo, R. S. L., \& Euzébios Filho, A. (2005). Desigualdade social e sistema educacional brasileiro: A urgência da educação emancipadora. Escritos sobre Educação, 4(2), 39-48.

Johnson, S. K., Tirrell, J. M., Callina, K. S., \&Weiner, M. B. (2018). Configurations of young peoples' important life goals and their associations with thriving. Research in Human Development, 15(2), 139-166. https://doi.org/10.1080/1 5427609.2018.1441576

Justo, J. S. (2005). O "ficar" na adolescência e paradigmas de relacionamento amoroso da contemporaneidade. Revista do Departamento de Psicologia - UFF,17(1), 61-77.https://doi.org/10.1590/S0104-80232005000100005

Lei no 13.415, de 16 de fevereiro de 2017. (2017, 17 fev.).http://www.planalto.gov.br/ccivil_03/_ato2015-2018/2017/ lei/L13415.htm

$\mathrm{Li}, \mathrm{Y}$. (2001). School engagement: What it is and why it is important for positive youth development. In R. M. Lerner, J. V. Lerner \& J. B. Benson (Eds.), Advances in Child Development and Behavior (pp. 131-160). Elsevier. 
McKnight, P. E., \& Kashdan, T. B. (2009). Purpose in Life as a System that Creates and Sustains Health and WellBeing: An Integrative, Testable Theory. Review of General Psychology, 13(3), 242-251. https://doi.org/10.1037/ a0017152

Pereira, B. C., Gobbo, J. P., \& Dellazzana-Zanon, L. L. (2017). Purpose in life scale for adolescents: Item construction process (EPVA) [Trabalho apresentado]. III International Symposium Adolescence(S) \& I Fórum (Re)Pensando a Educação (pp. 370-380). http://doi.org/10.22388/2525-5894.2017.30

Riter, H. S., Dellazzana-Zanon, L. L., \& Freitas, L. B. D. L. (2019). Projetos de vida de adolescentes de nível socioeconômico baixo quanto aos relacionamentos afetivos, Revista da SPAGESP, 20(1), 55-68.

Sallas, A. L. F. (1999). Os jovens de Curitiba: Desencantos e esperanças: Juventude, violência e cidadania. Unesco.

Sarriera, J. C., Silva, M. A., Kabbas, C. P., \& Lópes, V. B. (2001). Formação da identidade ocupacional em adolescentes. Estudos de Psicologia, 6(1), 27-32. https://doi.org/10.1590/S1413-294X2001000100004

Serrão, M., \& Baleeiro, M. C. (1999). Aprendendo a ser e a conviver (2a ed.). FTD.

Sousa, F. F. V., Nunes, M. L. S., \& Machado, C. J. S. (2012). “Ficar é...”: um código de relacionamento entre adolescentes. Caderno Espaço Feminino, 25(2), 136-157.

Stevenson, D. J., \& Baker, D. P. (1987). The Family School Relation and the Child's School Performance. Child Development, 58, 1348-1357. https:// doi.org/10.2307/1130626

Teixeira, E. J. (2005). Juventude pobre, participação e redes de sociabilidade na construção do projeto de vida [Dissertação de mestrado não-publicada, Universidade Federal do Rio de Janeiro].

Valore, L. A., \&Viaro, R.V. (2007). Profissão e sociedade no projeto de vida de adolescentes em orientação profissional. Revista Brasileira de Orientação Profissional, 8(2), 57-70.

Zonta, A. G. (2007). A construção do projeto de vida do aluno da rede pública de educação. Psicologia e Argumento, 25(50), 259-266.

\section{Bruna Caroline Pereira}

Mestre em Psicologia pela Pontifícia Universidade Católica de Campinas (PUC-Campinas), Campinas - SP. Brasil.

E-mail: bruna_caroline_2@hotmail.com

(1) https://orcid.org/0000-0002-1583-8274

\section{Cristian Zanon}

Psicólogo, Mestre, Doutor e Pós-Doutor em Psicologia. Professor Adjunto de Métodos Quantitativos do Programa de Pós-Graduação em Psicologia na Universidade Federal do Rio Grande do Sul (UFRGS), Porto Alegre - RS. Brasil.

E-mail: cristian.zanon@ufrgs.br

(D) https://orcid.org/0000-0003-3822-5275

\section{Letícia Lovato Dellazzana-Zanon}

Docente do Programa de Programa de Pós-Graduação em Psicologia na Pontifícia Universidade Católica de Campinas (PUC-Campinas), Campinas - SP. Brasil.

E-mail: leticiadellazzana@gmail.com

(1) https://orcid.org/0000-0003-0649-1675

Endereço para envio de correspondência:

Programa de Pós-Graduação em Psicologia. Av. John Boyd Dunlop, s/n, Bairro Jd. Ipaussurama. CEP: 13060-904, Campinas - SP. Brasil.

Recebido 26/08/2019

Aceito 05/11/2020 
Psicologia: Ciência e Profissão 2021 v. 41, e227915-1-14.

Received 08/26/2019

Approved 11/05/2020

Recibido 26/08/2019

Aceptado 05/11/2020

Como citar: Pereira, B. C., Zanon, C., \& Dellazzana-Zanon, L. L. (2021). Influência dos contextos escolar e familiar nos projetos de vida de adolescentes. Psicologia: Ciência e Profissão, 41, 1-14. https://doi.org/10.1590/1982-

3703003227915

How to cite: Pereira, B. C., Zanon, C., \& Dellazzana-Zanon, L. L. (2021). Influence of School and Family Contexts in Adolescents' Life Purposes. Psicologia: Ciência e Profissão, 41, 1-14. https://doi.org/10.1590/1982-3703003227915

Cómo citar: Pereira, B. C., Zanon, C., \& Dellazzana-Zanon, L. L. (2021). Influencia de los Contextos Escolar y Familiar en los Proyectos de Vida de Adolescentes. Psicologia: Ciência e Profissão, 41, 1-14. https://doi.org/10.1590/

1982-3703003227915 